\title{
Nomogram for predicting post-traumatic hydrocephalus after decompressive craniectomy for traumatic brain injury
}

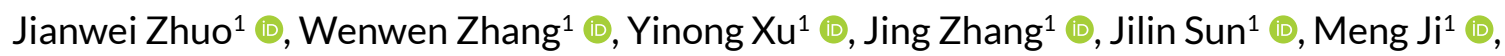 \\ Kai Wang ${ }^{1}$,, Yuhai Wang ${ }^{2 *}$ (1)
}

\begin{abstract}
SUMMARY
OBJECTIVE: This study aimed to develop and validate a practical nomogram to predict the occurrence of post-traumatic hydrocephalus in patients who have undergone decompressive craniectomy for traumatic brain injury.

METHODS: A total of 516 cases were enrolled and divided into the training $(n=364)$ and validation $(n=152)$ cohorts. Optimal predictors were selected through least absolute shrinkage and selection operator regression analysis of the training cohort then used to develop a nomogram. Receiver operating characteristic, calibration plot, and decision curve analysis, respectively, were used to evaluate the discrimination, fitting performance, and clinical utility of the resulting nomogram in the validation cohort.

RESULTS: Preoperative subarachnoid hemorrhage Fisher grade, type of decompressive craniectomy, transcalvarial herniation volume, subdural hygroma, and functional outcome were all identified as predictors and included in the predicting model. The nomogram exhibited good discrimination in the validation cohort and had an area under the receiver operating characteristic curve of $0.80(95 \% \mathrm{Cl} 0.72-0.88)$. The calibration plot demonstrated goodness-of-fit between the nomogram's prediction and actual observation in the validation cohort. Finally, decision curve analysis indicated significant clinical adaptability.

CONCLUSION: The present study developed and validated a model to predict post-traumatic hydrocephalus. The nomogram that had good discrimination, calibration, and clinical practicality can be useful for screening patients at a high risk of post-traumatic hydrocephalus. The nomogram can also be used in clinical practice to develop better therapeutic strategies.

KEYWORDS: Post-traumatic hydrocephalus. Decompressive craniectomy. Traumatic brain injury. Nomogram. Prediction model.
\end{abstract}

\section{INTRODUCTION}

Post-traumatic hydrocephalus (PTH) is one of the most important postoperative complications after decompressive craniectomy (DC) and is a major challenge to both patients with traumatic brain injury (TBI) and surgeons ${ }^{1}$. PTH is among the secondary insults aggravating brain damage and is characterized by impaired secretion, circulation, and malabsorption of the cerebrospinal fluid (CSF), resulting in ventricular dilatation ${ }^{2}$. The specific etiological mechanism of PTH has yet to be fully elucidated, which makes its early diagnosis and treatment difficult. PTH clearly impairs brain metabolism as well as function and often slows down the clinical improvement of patients. The condition also causes adverse outcomes if it is not promptly detected and managed ${ }^{3}$. Current researches have largely focused on exploring the risk factors, hence restricting their clinical application. Consequently, the present study developed and validated a nomogram to assist in predicting the risk of PTH in TBI patients who have undergone DC. The nomogram may therefore contribute to the timely and convenient identification of patients at a high risk of PTH and facilitate earlier clinical intervention.

\section{METHODS}

\section{Subjects}

The study included a total of 516 individuals out of the 584 TBI patients who had undergone DC between January 2009 and June 2020, at the neurosurgery department of the People's Liberation Army Joint Logistic Support Force 904th Hospital, Wuxi, Jiangsu, China. Later, the dataset was randomly partitioned into the training (364 patients) and validation (152 patients) cohorts in a ratio of 7:3. The study was approved by the ethics committee of the hospital. The following inclusion criteria

\footnotetext{
${ }^{1}$ The Fourth People's Hospital of Taizhou, Department of Neurosurgery - Taizhou, China.

2People's Liberation Army Joint Logistic Support Force 904th Hospital, Department of Neurosurgery - Wuxi, China.

*Corresponding author: yuhaiwang67@163.com

Conflicts of interest: the authors declare there is no conflicts of interest. Funding: none.

Received on July 22, 2021. Accepted on November 01, 2021.
} 
were used: (1) patients presenting with a definite history of craniocerebral trauma, confirmed through computed tomography (CT) scans in all the cases and received treatment according to the guidelines for severe $\mathrm{TBI}^{4}$; (2) those aged $\geq 18$ years; and (3) a minimum follow-up duration of 6 months. The following exclusion criteria were used: (1) patients with history of ventriculomegaly, meningitis, or malignancy in the nervous system; (2) those with other serious systemic diseases, including infections, cardiopulmonary failure, or hepatorenal insufficiency; (3) patients who died within 3 days of admission; (4) those with severe multiple injuries affecting cerebral blood perfusion; and (5) patients who were diagnosed with hydrocephalus before injury.

\section{Diagnostic criteria for post-traumatic hydrocephalus}

The diagnosis of PTH was done based on a combination of clinical characteristics and positive imaging results. The following characteristics were used: (1) neurological symptoms which included headache and vomiting, nerve deficits, and an altered level of consciousness; (2) the clinical state gradually improved over time but neurological deterioration and disturbance of consciousness worsened again; and (3) the brain CT scan showed progressive ventricular dilation (Evans index $>0.3)^{5}$.

\section{Data collection}

All the data are highlighted in Table 1. The data consisted of: (1) physiological characteristics including gender and age; (2) chronic conditions including hypertension and diabetes mellitus; (3) preoperative CT findings including the Fisher grade of subarachnoid hemorrhage (SAH), intraventricular hemorrhage (IVH), and midline shifting; (4) postoperative CT findings including type of DC, transcalvarial herniation volume (TCHV), subdural hygroma (SDG), and the craniectomy area. For the collection of postoperative radiological data, there is no fixed time period, but the principle of its maximum extent regardless the delay from surgery. The volume of transcalvarial herniation $(\mathrm{TCH})$ was calculated using a formula previously published by Liao et al. ${ }^{6}$ Notably, this formula has the advantages of having a simple principle, few parameters, and ease of implementation. Moreover, SDG was defined as low density, local, and non-lateral restricted regions of subdural CSF accumulation ${ }^{7}$; and (5) other potential factors including open-head injury, cause of head injury, preoperative GCS score, intracranial infection, and functional outcome. The functional outcome of each patient was measured according to the GOS score, 6 months post-discharge ${ }^{8}$. The outcomes were categorized into favorable (GOS 4 and 5) and unfavorable (GOS 1-3) based on the GOS scores ${ }^{1}$.

\section{Statistical analysis}

Variables that conformed to normal distribution were analyzed using the independent sample t-test and expressed as the meanl distribution were analyzed using the independenting to the GOS score, 6 months post-discharg-Whitney $\mathrm{U}$ test and expressed as the median (interquartile range). Classification variables were analyzed using the chi-square test or Fisher exact test, and given as proportions (percentages). Moreover, the least absolute shrinkage and selection operator (LASSO) method was used to identify the optimal features. Following this, the selected features used to develop a regression model. Afterwards, the model was transformed into a nomogram. In addition, the receiver operating characteristic (ROC) curve, calibration plot, and decision curve analysis (DCA), respectively, were used to assess the model. Data analysis was conducted using SPSS (version 26.0; IBM Corporation, Armonk, NY, USA), R (version 3.5.1; http:// www.r-project.org), and Stata (version 14.0; StataCorp, College Station, TX, USA).

\section{RESULTS}

\section{Clinical characteristics}

The clinical characteristics of patients in the training and validation cohorts are summarized in Table 1 . Out of the 364 patients in the training cohort, 98 (26.9\%) were diagnosed with PTH and the mean age was 46.6 years. In contrast, $41(27.0 \%)$ out of the 152 patients in the validation cohort were diagnosed with PTH and the mean age was 46.9 years.

\section{Results from feature selection and nomogram construction}

Least absolute shrinkage and selection operator regression was performed to screen out the nonzero features from the training cohort. Consequently, the partial likelihood deviance curve with the $\log$ (lambda) was plotted. In addition, two dotted vertical lines were drawn at the optimal values by determining the minimum criteria and the 1 standard error (SE) of the minimum criteria (Figure 1A). The number of potential predictors reduced from 16 to 5 (Figure 1B). Thereafter, the above-mentioned 5 unbiased variables, including preoperative SAH Fisher grade, type of DC, TCHV, SDG, and functional outcome, were used to build a model to predict the occurrence of PTH and displayed as a nomogram (Figure 2A). Moreover, it was applied by summing the points determined on the points scale, for each predictor. 
Table 1. Demographics and clinical characteristics of study in the training and validation cohorts.

\begin{tabular}{|c|c|c|c|c|c|c|}
\hline \multirow[b]{2}{*}{ Characteristic } & \multicolumn{2}{|c|}{ Training cohort } & \multirow[b]{2}{*}{$\mathbf{p}$} & \multicolumn{2}{|c|}{ Validation cohort } & \multirow[b]{2}{*}{$\mathbf{p}$} \\
\hline & $\begin{array}{c}\text { Non-PTH } \\
(n=266)\end{array}$ & PTH (n=98) & & $\begin{array}{c}\text { Non-PTH } \\
(n=111)\end{array}$ & PTH $(n=41)$ & \\
\hline \multicolumn{7}{|l|}{ Gender, n (\%) } \\
\hline Male & $184(69.2)$ & $73(74.5)$ & \multirow{2}{*}{0.323} & $88(79.3)$ & $31(75.6)$ & \multirow{2}{*}{0.626} \\
\hline Female & $82(30.8)$ & $25(25.5)$ & & $23(20.7)$ & $10(24.4)$ & \\
\hline Age (year), mean $\pm S D$ & 45.71 & 49.18 & 0.047 & 47.54 & 45.32 & 0.413 \\
\hline \multicolumn{7}{|l|}{ Open-head injury, n (\%) } \\
\hline No & $229(86.1)$ & $83(84.7)$ & \multirow{2}{*}{0.736} & $96(86.5)$ & $32(78.0)$ & \multirow{2}{*}{0.205} \\
\hline Yes & $37(13.9)$ & 15 (15.3) & & $15(13.5)$ & $9(22.0)$ & \\
\hline \multicolumn{7}{|l|}{ Head injury cause, n (\%) } \\
\hline Traffic accident & $130(48.9)$ & $60(61.2)$ & \multirow{4}{*}{0.160} & $56(50.5)$ & $19(46.3)$ & \multirow{4}{*}{0.482} \\
\hline Fall down & $20(7.5)$ & $8(8.2)$ & & $10(9.0)$ & $5(12.2)$ & \\
\hline Slip down & $48(18.0)$ & $12(12.2)$ & & 20(18.0) & $11(26.8)$ & \\
\hline Others & $68(25.6)$ & $18(18.4)$ & & $25(22.5)$ & $6(14.7)$ & \\
\hline \multicolumn{7}{|l|}{ Hypertension, n (\%) } \\
\hline No & $225(84.6)$ & $75(76.5)$ & \multirow{2}{*}{0.073} & $83(74.8)$ & $34(82.9)$ & \multirow{2}{*}{0.289} \\
\hline Yes & $41(15.4)$ & $23(23.5)$ & & $28(25.2)$ & $7(17.1)$ & \\
\hline \multicolumn{7}{|l|}{ Diabetes mellitus, n (\%) } \\
\hline No & $253(95.1)$ & $91(92.9)$ & \multirow{2}{*}{0.402} & $107(96.4)$ & 39 (95.1) & \multirow{2}{*}{0.661} \\
\hline Yes & $13(4.9)$ & $7(7.1)$ & & $4(3.6)$ & $2(4.9)$ & \\
\hline \multicolumn{7}{|c|}{ Preoperative GCS score, n (\%) } \\
\hline$\leq 8$ & $148(55.6)$ & $68(69.4)$ & \multirow{2}{*}{0.018} & $62(55.9)$ & $29(70.7)$ & \multirow{2}{*}{0.097} \\
\hline$>8$ & $118(44.6)$ & $30(30.6)$ & & $49(44.1)$ & $12(29.3)$ & \\
\hline \multicolumn{7}{|c|}{ Preoperative SAH Fisher grade, n (\%) } \\
\hline 1 & $110(41.4)$ & $23(23.5)$ & \multirow{4}{*}{$<0.001$} & $44(39.6)$ & $7(17.1)$ & \\
\hline 2 & $85(32.0)$ & $22(22.4)$ & & $31(27.9)$ & $12(29.3)$ & O18 \\
\hline 3 & $43(16.2)$ & $31(31.6)$ & & $21(18.9)$ & $9(22.0)$ & 0.010 \\
\hline 4 & $28(10.5)$ & $22(22.4)$ & & $15(13.5)$ & $13(31.6)$ & \\
\hline IVH, n (\%) & & & & & & \\
\hline No & $250(94.0)$ & $85(86.7)$ & ק حمि & $107(96.4)$ & $35(85.4)$ & \\
\hline Yes & $16(6.0)$ & $13(13.3)$ & 0.023 & $4(3.6)$ & $6(14.6)$ & 0.024 \\
\hline Midline shifting (cm), $\mathrm{n}(\%)$ & & & & & & \\
\hline$<0.5$ & $85(32.0)$ & $20(20.4)$ & & $28(25.2)$ & $9(22.0)$ & \\
\hline $0.5-1$ & $113(42.5)$ & $42(42.9)$ & 0.040 & $49(44.2)$ & $22(53.6)$ & 0.573 \\
\hline$>1$ & $68(25.6)$ & $36(36.7)$ & & $34(30.6)$ & $10(24.4)$ & \\
\hline Type of DC, n (\%) & & & & & & \\
\hline Unilateral craniectomy & $228(85.7)$ & $66(67.3)$ & $<\cap \cap \cap 1$ & $99(89.2)$ & $28(68.3)$ & ?חח ח \\
\hline Bilateral craniectomy & $38(14.3)$ & $32(32.7)$ & -0.001 & $12(10.8)$ & $13(31.7)$ & 0.002 \\
\hline Intracranial infection, n (\% & & & & & & \\
\hline No & $226(85.0)$ & $68(69.4)$ & م००1 & $90(81.1)$ & $28(68.3)$ & 0093 \\
\hline Yes & $40(15.0)$ & $30(30.6)$ & 0.001 & $21(18.9)$ & $13(31.7)$ & 0.093 \\
\hline
\end{tabular}


Table 1. Continuation.

\begin{tabular}{|c|c|c|c|c|c|c|}
\hline \multirow[b]{2}{*}{ Characteristic } & \multicolumn{2}{|c|}{ Training cohort } & \multirow[b]{2}{*}{ p } & \multicolumn{2}{|c|}{ Validation cohort } & \multirow[b]{2}{*}{$\mathbf{p}$} \\
\hline & $\begin{array}{c}\text { Non-PTH } \\
(n=266)\end{array}$ & PTH (n=98) & & $\begin{array}{c}\text { Non-PTH } \\
(n=111)\end{array}$ & PTH (n=41) & \\
\hline $\mathrm{TCHV}\left(\mathrm{cm}^{3}\right)$, median (IQR) & $46(36.0-57.0)$ & $69(50.0-93.0)$ & $<0.001$ & $49(33.0-63.0)$ & $69(48.5-94.5)$ & $<0.001$ \\
\hline \multicolumn{7}{|l|}{ SDG, n (\%) } \\
\hline No & $166(62.4)$ & $43(43.9)$ & \multirow{5}{*}{$<0.001$} & $76(68.5)$ & $11(26.8)$ & \multirow{5}{*}{$<0.001$} \\
\hline Ipsilateral & $62(23.3)$ & $15(15.3)$ & & $16(14.4)$ & $12(29.3)$ & \\
\hline Contralateral & $8(3.0)$ & $4(4.1)$ & & $6(5.4)$ & $2(4.9)$ & \\
\hline Bilateral & $23(8.7)$ & $21(21.4)$ & & $9(8.1)$ & $13(31.7)$ & \\
\hline Interhemispheric & $7(2.6)$ & $15(15.3)$ & & $4(3.6)$ & $3(7.3)$ & \\
\hline Craniectomy area $\left(\mathrm{cm}^{2}\right)$, median (IQR) & $\begin{array}{c}89.7(79.9- \\
111.1)\end{array}$ & $\begin{array}{c}102.2(88.9- \\
137.1)\end{array}$ & $<0.001$ & $\begin{array}{c}88.0(78.5- \\
106.8)\end{array}$ & $\begin{array}{c}107.1 \text { (89.4- } \\
144.0)\end{array}$ & $<0.001$ \\
\hline \multicolumn{7}{|l|}{ Functional outcome, n (\%) } \\
\hline Unfavorable & $67(25.2)$ & $62(63.3)$ & \multirow{2}{*}{$<0.001$} & $32(28.8)$ & $21(51.2)$ & \multirow{2}{*}{0.010} \\
\hline Favorable & $199(74.8)$ & $36(36.7)$ & & $79(71.2)$ & $20(48.8)$ & \\
\hline
\end{tabular}

PTH: post-traumatic hydrocephalus; SD: standard deviation; GCS: Glasgow Coma Scale; SAH: subarachnoid hemorrhage; IVH: intraventricular hemorrhage; DC: decompressive craniectomy; TCHV: transcalvarial herniation volume; SDG: subdural hygroma; IQR: interquartile range.

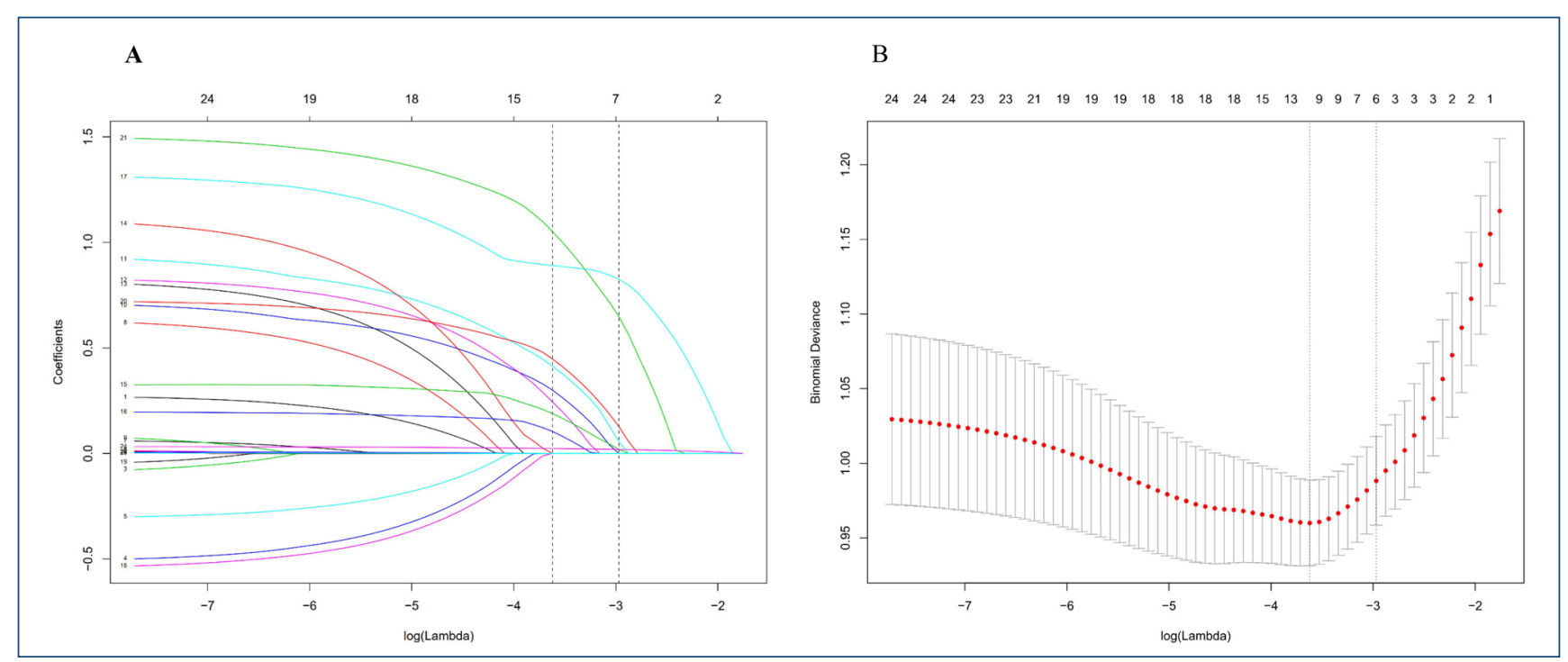

Figure 1. Selection of predictor features in the training cohort using least absolute shrinkage and selection operator regression. (A) The selected optimal parameter (lambda) in the least absolute shrinkage and selection operator model was 10-fold cross-validation based on the minimum criteria. (B) Least absolute shrinkage and selection operator coefficient profiles of the 16 characteristics. Five features with nonzero coefficients were finally obtained. LASSO: least absolute shrinkage and selection operator; SE: standard error.

\section{Validation of the nomogram}

The ROC curve for the validation cohort was generated by plotting the true positivity rate (y-axis) against the false positivity rate ( $\mathrm{x}$-axis) and bold black solid curve represents the discriminatory ability of the nomogram. In addition, Figure 2B demonstrates that the prediction model had a fairly good discriminatory ability, with an area under the ROC curve of 0.80
(95\%CI 0.72-0.88). Moreover, the calibration curve was generated by plotting the actual diagnosed PTH (y-axis) against the predicted incidence risk (x-axis). The results in Figure 2C show a good fitting degree between prediction and observation.

Furthermore, DCA on the validation cohort showed that the capacity of the nomogram to predict the occurrence of PTH was more beneficial than either the "treat-all" or "treat-none" 
A

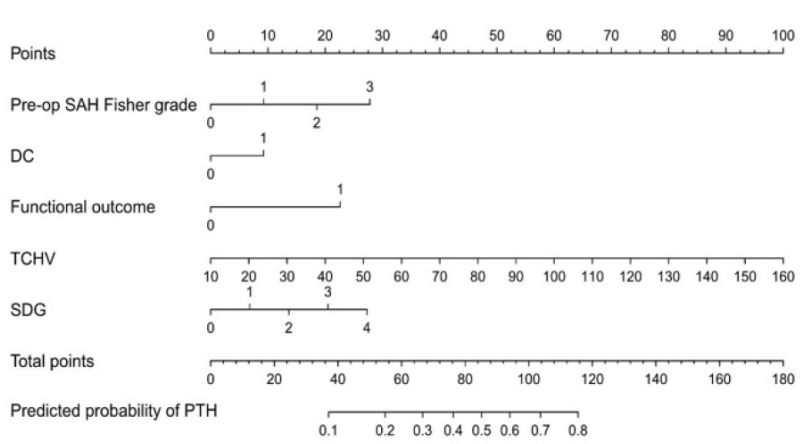

C

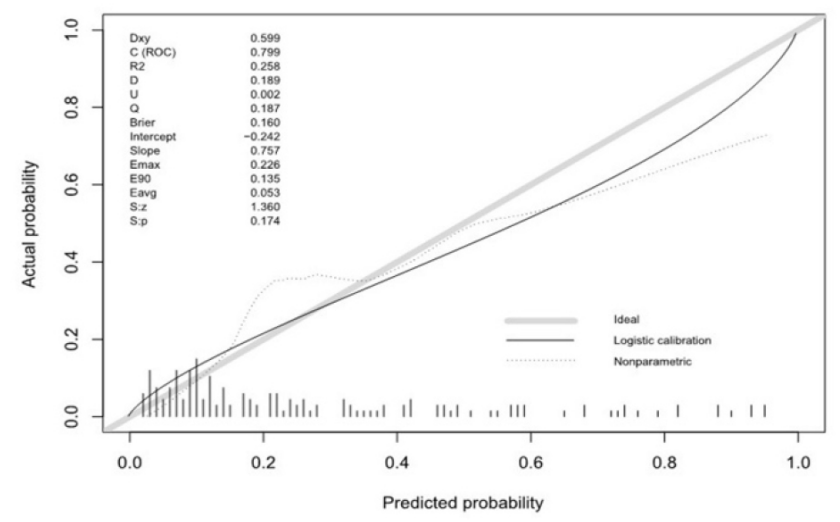

B

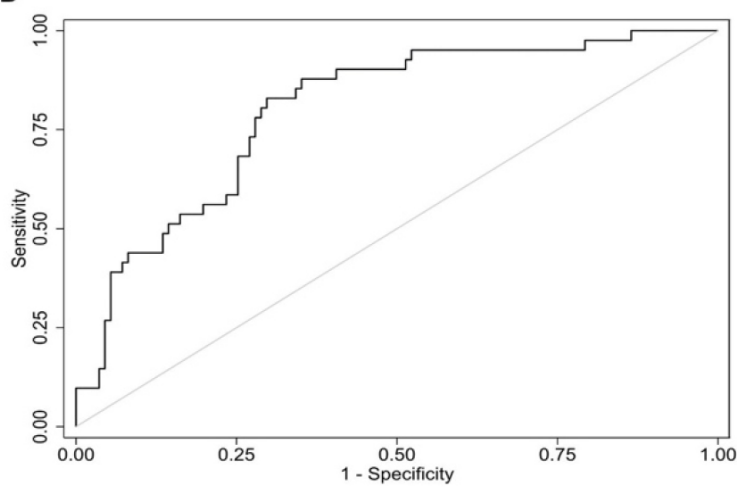

D

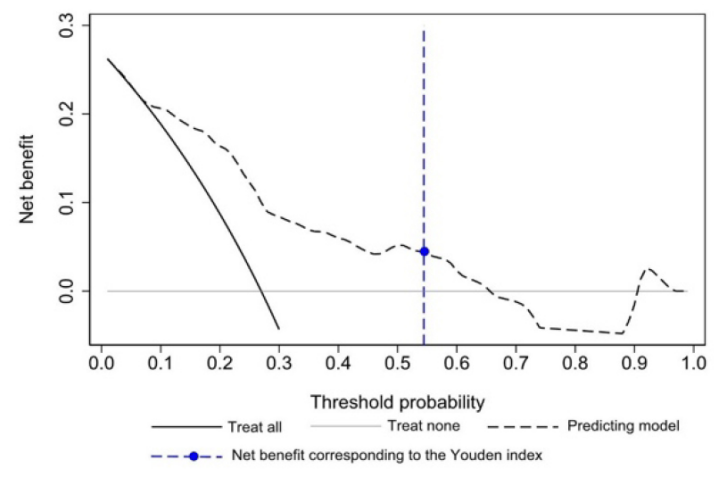

Figure 2. Development and validation of a predictive model for post-traumatic hydrocephalus. (A) A nomogram consisting of the preoperative subarachnoid hemorrhage Fisher grade, type of decompressive craniectomy, transcalvarial herniation volume, subdural hygroma, and functional outcome. Draw a line perpendicular from the corresponding axis of each predictor until it reaches the top line labeled "Points". Sum up the number of points for all predictors then draw a line descending from the axis labeled "Total points" until it intercepts the predicted probability of post-traumatic hydrocephalus axes to determine probabilities of post-traumatic hydrocephalus. For pre-op subarachnoid hemorrhage Fisher grade, $0=$ =Fisher 1 grade, 1=Fisher 2 grade, 2=Fisher 3 grade, $3=$ Fisher 4 grade. For decompressive craniectomy, $0=$ unilateral, $1=$ bilateral. For functional outcome, $0=$ unfavorable, $1=$ favorable. For subdural hygroma, $0=$ no subdural hygroma, $1=$ ipsilateral, $2=$ contralateral, $3=$ bilateral, 4=interhemispheric. (B) A receiver operating characteristic curve to evaluate the discriminating capability of the nomogram. (C) A calibration plot to evaluate the fitting performance of the nomogram. (D) Decision curve analysis to evaluate the clinical utility of the nomogram. SAH: subarachnoid hemorrhage; DC: decompressive craniectomy; TCHV: transcalvarial herniation volume; SDG: subdural hygroma; ROC: receiver operating characteristic; DCA: decision curve analysis.

strategy, with a threshold probability of $8-66 \%$ (Figure 2D). Additionally, the DCA results of the model in the validation cohort showed that there was a satisfactory net clinical benefit even under the Youden index (0.532).

\section{DISCUSSION}

Although increasing clinical attention has recently been paid to PTH, early, and reliable prediction of PTH using available clinical evidence is challenging. Therefore, it is necessary to develop methods that enable early diagnosis of PTH in order to improve decision-making in clinical practice. In the present study, demographic, clinical, and neuroimaging data from $\mathrm{PTH}$ patients were analyzed to examine the association between the putative risk factors. The study used LASSO regression to develop a nomogram for predicting the incidence of $\mathrm{PTH}$. The statistical results herein indicated that the model had a satisfactory goodness-of-fit, robustness, and predictive ability. Therefore, the model could be of great significance in effectively predicting and preventing the progression of PTH. In this study, 16 factors that may have been related to the occurrence of PTH were analyzed through LASSO regression and 5 predictors were finally obtained. The nomogram showed that the weight of all the predictors could be introduced into the model intuitively and visually.

The nomogram showed that PTH was closely related to severity of traumatic SAH. Previous studies reported that the 
probability of PTH in patients with severe $\mathrm{SAH}$ was significantly higher than that in patients without or mild $\mathrm{SAH}^{9}$. One of the many theories proposed to explain the mechanisms underlying the occurrence of hydrocephalus after SAH is that SAH disturbs CSF circulation at the basal cisterns, ventricles, the foramen of Monro, or the extensive subarachnoid space ${ }^{10,11}$. In addition, Chen et al. ${ }^{3}$ showed that the Fisher 4 grade is among the strongest radiological factors affecting the occurrence of PTH.

Destruction of the integrity of the cranial cavity was shown to be a major cause of PTH in patients who had undergone DC. It is noteworthy that bilateral DC leads to the outward transmission of pressure pulses in the bilateral cranial cavity, leading to more serious disturbance of CSF circulation and high chances of $\mathrm{PTH}^{12}$. Moreover, TCH following DC occurred frequently. Furthermore, a study by Neto et al. ${ }^{13}$ similarly suggested that TCHV can act as a predictor of PTH after DC. $\mathrm{TCH}$ has a complex pathogenic mechanism, that is, intracranial hypertension caused by various factors leads to a critical reduction in cerebral perfusion, and the brain bulges from the skull defect after DC, leading to the so-called TCH. The drainage of veins and circulation of CSF in herniated brain tissue are blocked, further aggravating both edema in herniated brain tissue and incarceration, thus affecting prognosis and outcomes. From this point of view, it is possible that a large craniectomy area reduces the occurrence of TCH but also causes a series of problems including high infection rates, difficulties in wound healing, and challenges in skull reconstruction ${ }^{14}$. Therefore, the craniectomy area should not be undertaken haphazardly.

The patients who have been in a coma for extended periods of time after operation often experience severe damage to the brain tissue, and the CSF circulation and absorption balance are damaged to a greater extent correspondingly, making it conducive for the development of PTH. In the present study, the results showed that PTH patients had an obviously unfavorable functional outcome, suggesting that they were likely to experience more severe TBI. This was interesting, however since preoperative GCS was not independently associated with PTH, similar to previous studies ${ }^{15}$. It is possible that there was more death of patients with a low preoperative GCS score in the immediate and shot-term postoperative period, thus indirectly decreasing the occurrence of PTH.

Subdural hygroma was shown to be one of the phenomena affecting changes in the dynamics of CSF, and may emerge prior to the occurrence of PTH. Previous studies also suggested that SDG and PTH are essentially caused by disorders in CSF hydrodynamics ${ }^{9}{ }^{16}$. SDG may represent local damage of CSF homeostasis while PTH indicates a more serious CSF dysfunction. Therefore, patients with SDG have higher chances of developing $\mathrm{PTH}^{16}$. The results from the present study indicated that the probability of developing PTH in bilateral SDG and interhemispheric hygroma was significantly higher than that of developing PTH in ipsilateral or contralateral hygroma, corroborating with the findings previously reported by Ki et al. ${ }^{17}$ In addition, Kaen et al..$^{18}$ similarly showed that interhemispheric hygroma is an important imaging characteristic that predicts the occurrence of PTH.

Receiver operating characteristic curve and calibration plot validated the effectiveness of the model. DCA is a method of evaluating prediction models by calculating the clinical net benefit. The DCA results showed that all the patients benefited from this model, with a threshold probability ranging from 8 to $66 \%$. This further verified the value of the model in practical clinical work.

The study had a number of limitations. First, this was a retrospective study and may therefore have a certain degree of selection and analytical bias. Second, a limited number of patients were recruited from a single institution. Although the prediction model was verified using a validation set, it is necessary to conduct large sample and multicenter studies to prove the feasibility of the nomogram so as to increase the possibility of extensive popularization of the model.

\section{CONCLUSIONS}

The present study used demographic, clinical, and neuroimaging indicators to develop a model for assessing the risk of PTH after undergoing DC in TBI patients. The indicators included the SAH Fisher grade, type of DC, TCHV, SDG, and functional outcome. In addition, the ROC curve, calibration plot, and DCA were used to show that the nomogram had a good predictive performance, calibration, and clinical utility. Moreover, the nomogram had the qualities of concise composition with fewer variables and can therefore be used for the identification of individuals at a high risk of PTH so that timely intervention can be implemented.

\section{AUTHORS' CONTRIBUTIONS}

JwZ: Conceptualization, Data curation, Formal analysis, Investigation, Methodology, Software, Visualization, Writing original draft. WZ: Formal analysis, Investigation, Methodology, Project administration, Software, Visualization, Writing - original draft. YX: Conceptualization, Supervision. JZ: Project administration. JS: Investigation, Methodology. MJ: Methodology. KW: Supervision, Visualization. YW: Conceptualization, Data curation, Project administration, Resources, Supervision, Validation, Writing - review \& editing. JZ and WZ: contributed to this paper equally, both as the first authors. 


\section{REFERENCES}

1. SuTM, Lan CM, Lee TH, Hsu SW, Tsai NW, Lu CH. Risk factors for the development of posttraumatic hydrocephalus after unilateral decompressive craniectomy in patients with traumatic brain injury. J Clin Neurosci. 2019;63:62-7. https://doi.org/10.1016/j. jocn.2019.02.006

2. Shim JW, Territo PR, Simpson S, Watson JC, Jiang L, Riley AA, et al. Hydrocephalus in a rat model of Meckel Gruber syndrome with a TMEM67 mutation. Sci Rep. 2019;9(1):1069. https://doi. org/10.1038/s41598-018-37620-5

3. Chen H, Yuan F, Chen SW, Guo Y, Wang G, Deng ZF, et al. Predicting posttraumatic hydrocephalus: derivation and validation of a risk scoring system based on clinical characteristics. Metab Brain Dis. 2017;32(5):1427-35.https://doi.org/10.1007/s11011-017-0008-2

4. Garringer JA, Niyonkuru C, McCullough EH, Loucks T, Dixon CE, Conley YP, et al. Impact of aromatase genetic variation on hormone levels and global outcome after severe TBI. J Neurotrauma. 2013;30(16):1415-25. https://doi.org/10.1089/neu.2012.2565

5. Ozoner B, Kilic M, Aydin L, Aydin S, Arslan YK, Musluman AM, et al. Early cranioplasty associated with a lower rate of post-traumatic hydrocephalus after decompressive craniectomy for traumatic brain injury. Eur J Trauma Emerg Surg. 2020;46(4):919-26. https:// doi.org/10.1007/s00068-020-01409-x

6. Liao CC, Tsai YH, Chen YL, Huang KC, Chiang IJ, Wong JM, et al. Transcalvarial brain herniation volume after decompressive craniectomy is the difference between two spherical caps. Med Hypotheses. 2015;84(3):183-8. https://doi.org/10.1016/j. mehy.2014.12.018

7. Lu VM, Carlstrom LP, Perry A, Graffeo CS, Domingo RA, Young $\mathrm{CC}$, et al. Prognostic significance of subdural hygroma for posttraumatic hydrocephalus after decompressive craniectomy in the traumatic brain injury setting: asystematic reviewand meta-analysis. Neurosurg Rev. 2021;44(1):129-38. https://doi.org/10.1007/ s10143-019-01223-z

8. Jennett B, Bond M. Assessment of outcome after severe brain damage: a practical scale. Lancet. 1975;305(7905):480-4. https:// doi.org/10.1016/s0140-6736(75)92830-5

9. Mohd Nor MA, Abdul Rahman NA, Adnan JS. Post-traumatic hydrocephalus. Malays J Med Sci. 2013;20(1):95-6. PMID:23613662
10. Lu X, Ji C, Wu J, You W, Wang W, Wang Z, et al. Intrathecal fibrinolysis for aneurysmal subarachnoid hemorrhage: evidence from randomized controlled trials and cohort studies. Front Neurol. 2019;10:885. https://doi.org/10.3389/fneur.2019.00885

11. Black PM, Tzouras A, Foley L. Cerebrospinal fluid dynamics and hydrocephalus after experimental subarachnoid hemorrhage. Neurosurgery. 1985;17(1):57-62. https://doi. org/10.1227/00006123-198507000-00009

12. Waziri A, Fusco D, Mayer SA, McKhann GM, Connolly ES Jr. Postoperative hydrocephalus in patients undergoing decompressive hemicraniectomy for ischemic or hemorrhagic stroke. Neurosurgery. 2007;61(3):489-94. https://doi.org/10.1227/01. NEU.0000290894.85072.37

13. Silva Neto AR, Uruguay ALR, Paiva DS, Silva ALP, Godeiro AHM, Eberlin LMN. Neurogenic bladder dysfunction as signal of late failure of endoscopic third ventriculostomy in child with spina bifida. World Neurosurg. 2019;128:454-7. https://doi.org/10.1016/j. wneu.2019.05.138

14. Weickenmeier J, Butler CAM, Young PG, Goriely A, Kuhl E. The mechanics of decompressive craniectomy: personalized simulations. Comput Methods Appl Mech Eng. 2017;314:180-95. https://doi. org/10.1016/j.cma.2016.08.011

15. Goldschmidt E, Deng H, Puccio AM, Okonkwo DO. Post-traumatic hydrocephalus following decompressive hemicraniectomy: incidence and risk factors in a prospective cohort of severe TBI patients. J Clin Neurosci. 2020;73:85-8. https://doi.org/10.1016/j. jocn.2020.01.027

16. De Bonis P, Sturiale CL, Anile C, Gaudino S, Mangiola A, Martucci M, et al. Decompressive craniectomy, interhemispheric hygroma and hydrocephalus: a timeline of events? Clin Neuro Neurosurg. 2013;115(8):1308-12. https://doi.org/10.1016/j. clineuro.2012.12.011

17. Ki HJ, Lee HJ, Lee HJ, Yi JS, Yang JH, Lee IW. The risk factors for hydrocephalus and subdural hygroma after decompressive craniectomy in head injured patients. J Korean Neurosurg Soc. 2015;58(3):254-61. https://doi.org/10.3340/jkns.2015.58.3.254

18. Kaen A, Jimenez-Roldan L, Alday R, Gomez PA, Lagares A, Alén JF, et al. Interhemispheric hygroma after decompressive craniectomy: does it predict posttraumatic hydrocephalus? J Neurosurg. 2010;113(6):1287-93. https://doi.org/10.3171/2010.4.JNS10132 